\title{
Automatic method based on image analysis for pilling evaluation in fabrics
}

\author{
Héctor C. Abril \\ María S. Millán, MEMBER SPIE \\ Universidad Politécnica de Cataluña \\ Departamento de Optica y Optometría \\ C/Violinista Vellsolà 37 \\ 08222 Terrassa \\ Barcelona, Spain \\ E-mail: abril@bec.oo.upc.es \\ Yezid Torres, MEMBER SPIE \\ Universidad Industrial de Santander \\ Laboratorio de Optica y Tratamiento \\ de Señales \\ AA 678 Bucaramanga \\ Santander, Colombia \\ Rafael Navarro \\ Instituto de Optica \\ Consejo Superior de Investigaciones \\ Científicas \\ C/Serrano 121 \\ 28006 Madrid \\ Spain
}

\begin{abstract}
A fully automatic method for pilling evaluation in wear-andtear fabrics is developed from the image analysis of a set of standard photographs (Zweigle KG-741 Reutlingen). The method involves operations in both the spatial and frequency domains to segment pills from the textured background of the web. It calculates the total area of pilling in the sample image and assigns a degree of pilling according to the standard. Two mathematical descriptions are analyzed according to the underlying rule established by the standard images, using the visual estimation of the area of pilling performed by a group of observers. A logarithmic (in base two) approach, which is consistent with human visual perception laws and facilitates an optimization of the method, is eventually adopted. (c) 1998 Society of Photo-Optical Instrumentation Engineers. [S0091-3286(98)02111-4]
\end{abstract}

Subject terms: pilling; web inspection; digital image processing; image segmentation.

Paper 980107 received Mar. 17, 1998; revised manuscript received July 16, 1998; accepted for publication Aug. 3, 1998.

This is a deep revision of two preliminary papers. The first was presented at the EUROPTO Symposium on Lasers, Optics and Vision for Productivity in Manufacturing I held in Besançon, France, June 1996, and it appeared (unrefereed) in SPIE Proceedings Vol. 2786. The second one was presented at the EUROPTO Symposium on Lasers and Optics in Manufacturing held in Munich, Germany, June 1997, and appeared (unrefereed) in SPIE Proceedings Vol. 3101.

\section{Introduction}

Pilling is an effect of wear and tear that considerably spoils the original appearance of a fabric. Pilling generation begins with a migration of fibers to the external part of yarns, so that fluffiness emerges on the web surface. Due to friction, this fluffiness gets entangled and forms pills that remain suspended from the web by long fibers. Because of wear, some pills fall off, causing the additional effect of loss of material. ${ }^{1,2}$ Web resistance to pilling is commonly tested in the laboratory by specific machines for pilling generation. A sample of the original fabric is fixed in the machine, in which wear is simulated by the action of brushes and abrasive materials. These machines are usually supplied with a standard reference consisting of photographs of samples with different degrees of pilling (Table 1). Experts with long experience and training in web inspection assign a degree of pilling by looking at the sample processed by the machine. Standard photographs offer a useful reference to experts for visual comparison with samples affected by pilling.

Apart from the high cost of these subjective methods based on a visual estimation of pilling by experts, they have the drawback of not being very precise. Objective procedures based, for instance, on cutting off, counting and weighing pills do exist, but they have a very limited use. An objective method based on height measurements using laser triangulation was also proposed for grading fabric pilling. ${ }^{3}$
Image processing techniques are widely used for characterizing and inspecting textured materials, ${ }^{4}$ in general, thus including textiles. ${ }^{5-8} \mathrm{An}$ interesting study of pilling evaluation by digital image analysis was carried out by Konda et al. ${ }^{9}$ Images of real samples were captured under near-tangential illumination, which gave images with good pill-to-background contrast that were then compared with standard images. The original image was binarized by applying a double threshold. The first threshold was determined by discriminant analysis and the second threshold was established to include only pills bigger than a given minimum. Although threshold selection was sometimes imprecise, it was a big step forward over conventional visual testing by experts and provided some basis for a deeper study.

In this paper, we develop a fully automatic method for pilling evaluation by digital analysis that estimates the total area of the sample covered by pills. The method is designed, tested and optimized using Zweigle KG-741 (Reutlingen) standard images as a reference. The analysis procedure sequentially combines operations in both the spatial and frequency domains to properly segment pills from the textured background of the web. A preliminary version of the method was reported in Ref. 10 and several improvements were introduced later. ${ }^{11}$

The analysis of the set of standard images enables us to approach empirically the underlying relationship between the total area of pilling and the degree of pilling attributed to the standard samples. In addition, our automatic method 
Table 1 Description of degrees of pilling.

\begin{tabular}{cl}
\hline \hline Degree & \multicolumn{1}{c}{ Description } \\
\hline 1 & $\begin{array}{l}\text { No differences from the original can be appreciated } \\
\text { (no pilling) }\end{array}$ \\
2 & $\begin{array}{l}\text { Light superficial fluffiness (slight pilling) } \\
3\end{array}$ \\
4 & $\begin{array}{l}\text { Moderate fluffiness and/or isolated well defined pills } \\
\text { Intense fluffiness and evident pilling (severe pilling) } \\
5\end{array}$ \\
\hline \hline
\end{tabular}

is compared in this work to human observers (assisted by computer). We show that the method obtains consistent results in the calculation of the total area of pilling with respect to the results obtained by the observers.

\section{Method}

The general procedure is presented in the flow diagram in Fig. 1. The first step is image acquisition. Experimental conditions for capturing real samples such as illumination, relative position of sample and camera, etc. must be fixed so that an image with a good pill-to-background contrast is obtained. Near-tangential illumination generally provides a good image, in which pills appear brightly against a dark background. ${ }^{9}$ We analyze a set of standard photographs that do not require a particular illumination setup to be captured and digitized. Since pills appear in black on a white piece of textile material in the standard photographs, we consider the acquired standard images with reverse contrast as input images for the sake of a straightforward application of the method to the analysis of real samples. Figure 2 shows a partial image of the standard sample corresponding to the degree of pilling 4 (Zweigle KG-741) with reverse contrast. The whole image was digitized with $512 \times 512$ pixels and 256 gray levels. The acquisition system was arranged so as to obtain a digital image where the frequency of the periodic plain web structure of the original standard image was half the Nyquist frequency. This means that a thread in the

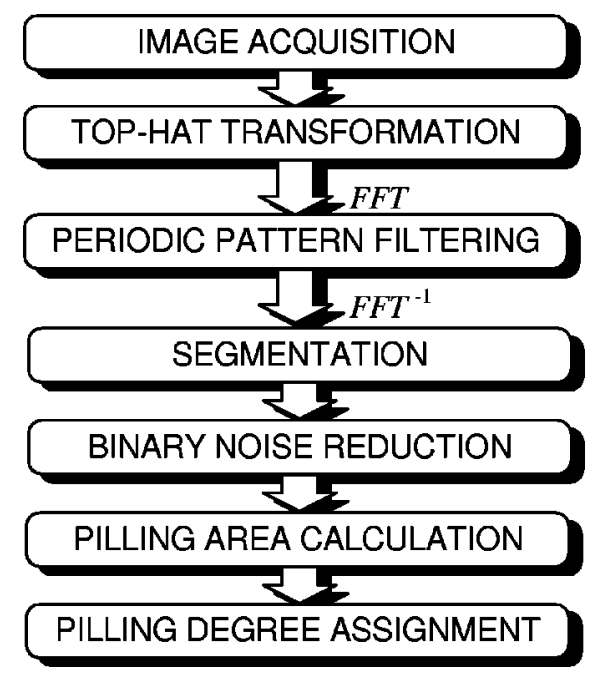

Fig. 1 Flow diagram of the method.

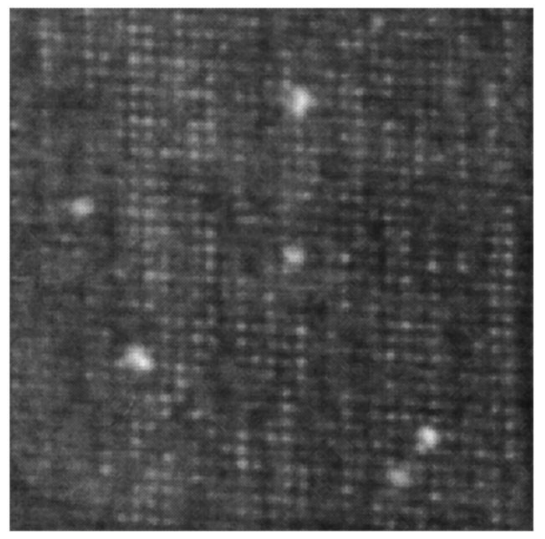

Fig. 2 Partial image of the standard sample corresponding to the degree of pilling 4 (Zweigle KG-741).

original standard image occupied 4 pixels on average. Because pill dimensions are commonly greater than the thread thickness, we considered that the acquired images with 4 pixels/thread had enough resolution. Big white blobs correspond to pills. The background exhibits the typical periodic structure of a textile web. A number of similar small white points regularly distributed throughout the image reveal this periodic structure.

The second step is a top-hat transform (Fig. 1), introduced to correct the input image for a possible nonuniform illumination. The third block was designed to isolate the randomly distributed white points of pilling from the periodically distributed small white points of the structure of the web. This is accomplished in the frequency domain by calculating the fast Fourier transform (FFT) of the image and then filtering out the peaks corresponding to periodic patterns. After an inverse Fourier transform, pills are segmented from the background in the filtered image at the fourth step of the method (segmentation, in Fig. 1). For this purpose we consider that the pixel histogram of the background can be modeled by a Gaussian distribution. This Gaussian model for the background is applied locally to the image to establish the threshold for each area of the image. The result is a binary image where objects (pills) are segmented from the background (now black). Noisy objects whose dimensions are too small to correspond to a pill are eliminated in the fifth step of the method. In the sixth step, the total area of pilling is obtained by adding together the pixels of the remaining objects, a measurement that must be related to the degree of pilling. From the areas of pilling of all the standard images, an empirical law on the area of pilling and the degree of pilling can be roughly worked out. A logarithmic function consistent with human vision features is used in the last stage of the method to assign a degree of pilling to a sample.

In Secs. 2.1 to 2.5 we describe in detail the steps in the method.

\subsection{Top-Hat Transform}

We apply a top-hat transform to make the background level low and uniform throughout the web image, thus compensating for a possible lack of uniformity in the illumination 


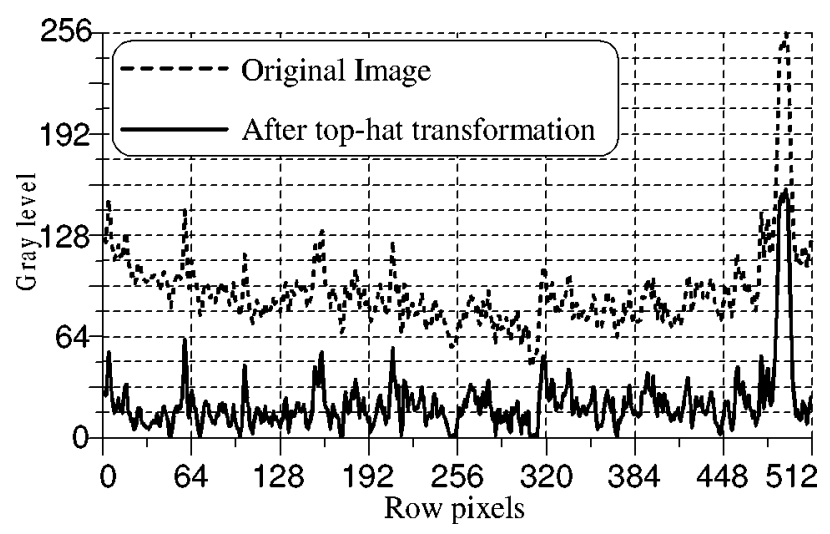

Fig. 3 Application of top-hat transform to a row of the standard image of degree of pilling $p=4$ (Zweigle KG-741).

of the sample. For a digitized image $I_{p}$ of a given sample with a degree of pilling $p$, the image after the top-hat transform $I_{p}^{\prime}$ is defined by ${ }^{12}$

$I_{p}^{\prime}=$ Top-hat $\left(I_{p}\right)=I_{p}-\left(I_{p} \circ g\right)$,

where $g$ is the kernel of the transformation and the symbol - indicates the gray-scale morphological opening operation. The kernel $g$ represents the maximum area of the image where an object (pill) could be contained, and thus its inner possible lack of uniformity is not altered. Edges and blobs are thus preserved and only the local average of the background level is subtracted from the image. Consequently, the kernel $g$ must be fixed at a slightly greater size than the largest pill of the image. As kernel $g$, we take a cylinder of 21 pixels diameter that is slightly larger than the largest pill found in all the standard images. Note that the size selection of kernel $g$ is not critical. In fact, there is not one unique kernel $g$ to obtain a reduction of the background level by the top-hat transform. The top-hat transform provides an increase in the pill-to-textile web contrast since it reduces the background level and preserves the gray-level differences between neighboring pixels.

As an example, Fig. 3 shows the result obtained by applying a top-hat transform to a row of the standard image of $p=4$. The profile in a dashed line corresponds to the original image. Its background is lower at the center than at the edges and has a high mean gray level of about 90. After applying a top-hat transform with the cylindrical kernel of 21 pixels diameter for $g$, the result is the profile presented in a solid line. Now the background level is much flatter and lower. The increase of the pill-to-background graylevel ratio can also be appreciated by looking at the pill represented by the peak on the right whose height is retained over a lower background level.

\subsection{Periodic Pattern Filtering}

In this step we aim to filter out all those bright points of the image that belong to the periodic structure of the web. These bright and relatively small points (see Fig. 2) could be confused with pills if they were not removed. We designed a filter to eliminate the periodic web structure that appears under the pills and in the image background. It is well known that the information corresponding to periodic structures in the spatial domain concentrates in high peaks at the fundamental and multiple frequencies of higher orders (harmonics) in the frequency domain. On the other hand, the Fourier transform of continuous images approaches zero with increasing frequency. For large values of frequency, the Fourier transform of an image having no gray-level discontinuities decreases in magnitude at least as fast as the inverse of the frequency. ${ }^{13}$

Furthermore, images of natural scenes tend to show a surprisingly homogeneous pattern of decay in the modulus of their Fourier spectra with the inverse of the frequency law. This law has been successfully considered in different applications such as in parametric blind deconvolution. ${ }^{14}$ For an image of a textile web affected by pilling, a mixture of these two distributions can be observed in the frequency domain. This mixture represents a rapid decay of the Fourier transform magnitude with frequency (according to the inverse of frequency law), corresponding to the nonperiodic patterns, together with a set of discrete and regularly distributed peaks that correspond to the periodic pattern. Let $|F(u, v)|$ be the magnitude of the Fourier transform (FT) of $I_{p}^{\prime},|F(u, v)|=\left|\mathrm{FT}\left[I_{p}^{\prime}(x, y)\right]\right|$. Except for the set of discrete and regularly distributed peaks, we can approximate $|F(u, v)|$ by the function:

$H(u, v)=\left(\frac{u^{2}}{a^{2}}+\frac{v^{2}}{b^{2}}\right)^{-1 / 2}$,

where $u$ and $v$ are the rectangular coordinates of the frequency domain, and $a$ and $b$ are real constants. Function $H(u, v)$ has elliptical symmetry around the center according to the two main, commonly orthogonal, directions of a web called weft and warp. In general, yarns of different brightness and width are woven with different densities in weft and warp directions, and so the fit in the frequency domain requires use of a function with elliptical symmetry. Obviously, the set of regularly distributed high peaks corresponding to the textile structure is not well fitted by the function of Eq. (2). But this fact can be exploited to block the information from the periodic pattern. Precisely because of this, we propose the application of a thresholding in the Fourier domain defined by:

$F^{\prime}(u, v)=\left\{\begin{array}{lll}F(u, v), & \text { for } & |F(u, v)|<k H(u, v) \\ 0, & \text { for } & |F(u, v)| \geqslant k H(u, v)\end{array}\right.$,

where $k$ is a real constant that satisfies $k>1$. The role of parameter $k$ is to control the height for making equal to 0 the peaks corresponding to the periodic pattern in the FT of the web sample. As an example, Fig. 4 represents by a dashed line a cross-section profile of the magnitude of the FT of the web sample of Fig. 2. We estimated $H(u, v)$ of Eq. (2) by a least squares fit of the entire distribution of the FT magnitude of the web sample (represented by a thin line in Fig. 4). The cross section of $k H(u, v)$ with $k=3.5$ is represented by a thick solid line in Fig. 4. All the values $F(u, v)$ with their magnitudes below $k H(u, v)$ remain unchanged after thresholding, whereas values whose magnitudes are equal to or higher than $k H(u, v)$ are canceled out. Most of the information corresponding to periodic patterns is thus removed, whereas most of the information corre- 


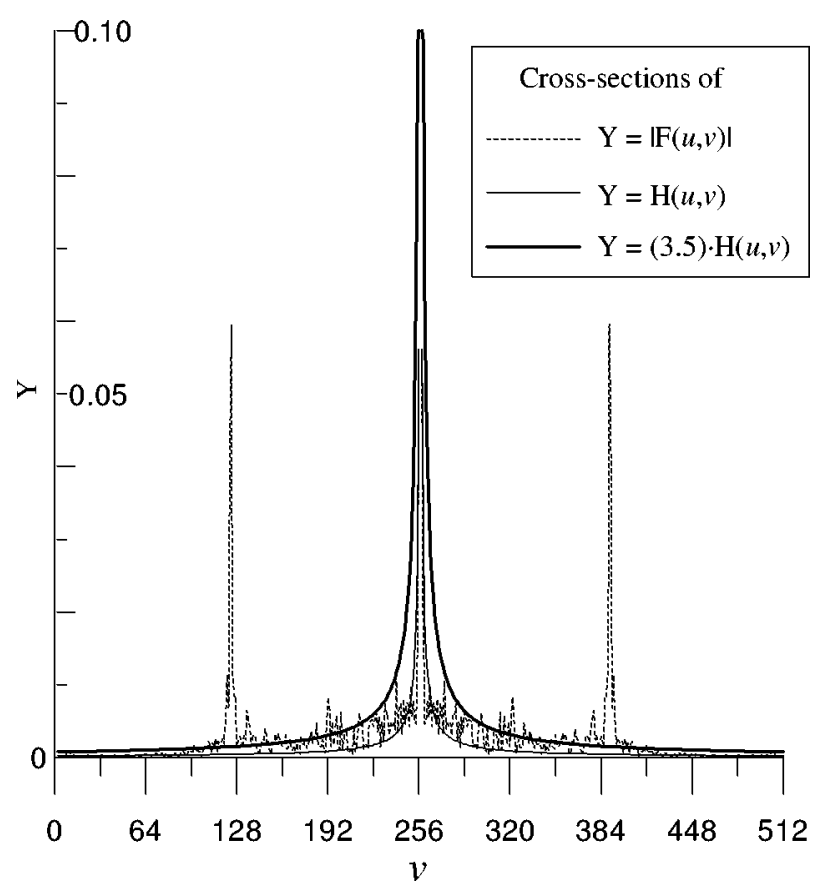

Fig. 4 Cross section of the mask proposed to filter the periodic pattern of the web image (thick solid line).

sponding to pills is preserved. The resulting filtered image in the spatial domain $I_{p}^{\prime \prime}$ is retrieved by applying the inverse FT to $F^{\prime}(u, v)$, that is $I_{p}^{\prime \prime}=\mathrm{FT}^{-1}\left[F^{\prime}(u, v)\right]$. Figure 5 shows the filtered image corresponding to Fig. 2 after tophat transformation and periodic pattern filtering with $k$ $=3.5$.

\subsection{Segmentation by Local Thresholding Based on a Gaussian Model for the Background Histogram}

Since a severe reduction of the bright small points belonging to the periodic pattern was achieved in the previous step, we assume that the dark area (consisting of pixels with low gray levels) in the filtered image corresponds to background, whereas various bright objects (consisting of

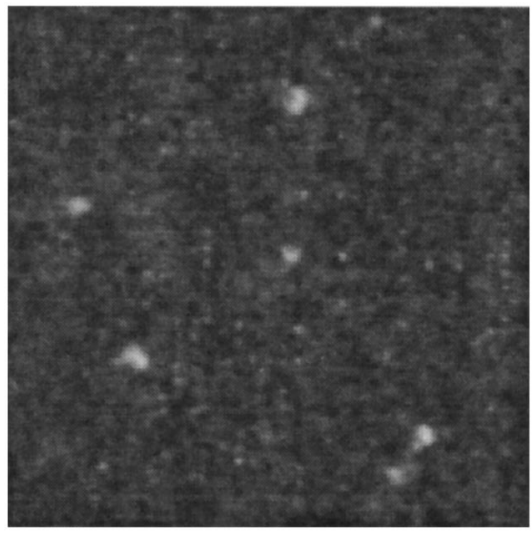

Fig. 5 Retrieved image after top-hat transformation and periodic pattern filtering with $k=3.5$.

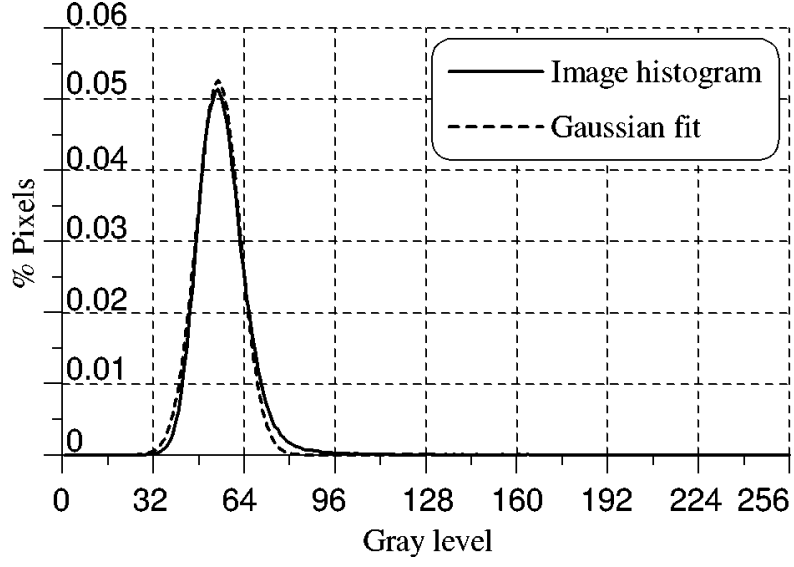

Fig. 6 Histogram of the entire image $I_{p}^{\prime \prime}$ (solid line), a sector of which is shown in Fig. 5, and its Gaussian fit (dashed line).

pixels with high gray levels) of different sizes and randomly distributed through the image correspond to pills.

In this step, we segment pills from the background to obtain a binary image in which white objects (pills) appear on a black background (web structure). In this particular case, the difficulty in establishing the threshold comes from the fact that the number of pixels belonging to the background can be several magnitude orders higher than the number of pixels belonging to pills.

The histogram of the image $I_{p}^{\prime \prime}$ (solid line in Fig. 6) shows only one peak placed at the dark part of the graylevel axis that clearly corresponds to the background. We assume that the background histogram can be approached by a symmetrical Gaussian distribution (dashed line in Fig. 6), so a threshold from the statistical parameters associated to that Gaussian can be established. Let $m$ and $\sigma$ be the respective mean value and standard deviation of the Gaussian obtained by a least squares fit of the image histogram. We propose a threshold value $U$ given by:

$U=m+N \sigma$,

where $N$ is a real positive parameter. For instance, if the Gaussian assumption was exact and we took $N=4$ in the threshold $U$ of Eq. (4), the expected misclassification percentage of background pixels (placed under Gaussian curve beyond the threshold) would be less than $0.003 \%$ of the rest of the background pixels placed under the Gaussian lobe. Nevertheless, since both classes, the background and pilling, have highly different numbers of pixels and our goal is to determine the number of pixels belonging to the pilling class, it would be more significant to refer these misclassified pixels to the number of pixels eventually considered as pilling. Thus, in the processed standard images we calculated the number of pixels under the Gaussian curve with gray levels higher than the threshold $U$ as a percentage of the total amount of pixels beyond this threshold. We repeated this calculation for three values of the parameter $N=3,4$, and 5 in the processed standard images of the five different degrees of pilling. Table 2 contains the results. For $N=3$, the mean error for the different degrees of pilling is around $6 \%$, whereas for $N=4$ it is $0.3 \%$ and for $N=5$ it 
Table 2 Error of misclassification in percent of background pixels in the total amount of pixels classified as pilling (beyond the threshold).

\begin{tabular}{clll}
\hline \hline & \multicolumn{3}{c}{ Error of Misclassification (\%) } \\
\cline { 2 - 4 } Degree of Pilling & $N=3$ & $N=4$ & $N=5$ \\
\hline 1 & 5.7 & 0.33 & 0.0073 \\
2 & 5.9 & 0.44 & 0.0131 \\
3 & 7.6 & 0.42 & 0.0073 \\
4 & 7.3 & 0.27 & 0.0045 \\
5 & 5.0 & 0.22 & 0.0030 \\
Mean error (\%) & 6 & 0.3 & 0.007 \\
\hline \hline
\end{tabular}

is $0.007 \%$. We take the value $N=4$ because it yields a reasonably low error of misclassification. For $N=5$ and higher the error is clearly much lower, but the resulting thresholds are too high. There is therefore a high probability that too many pixels of pilling would eventually be misclassified as background.

The application of the threshold given by Eq. (4) with $m$ and $\sigma$ values taken from the histogram of an entire image yields general binarization in the segmented image $I_{p}^{\prime \prime \prime}$. The result obtained with this binarization in the part of the standard image of pilling 4 of Fig. 2 is shown in Fig. 7. We investigate the possibility of further improving on this result by using local instead of global binarization. For this purpose, we define a square window of $M \times M$ pixels size that scans the image from left to right and from top to bottom. For a given window $i$ we calculate the local threshold $U_{i}$ in the same way as before, except for the histogram, which is now locally computed within the window $i$. Thus, the local threshold is given by:

$U_{i}=m_{i}+N \sigma_{i}$

where $m_{i}$ and $\sigma_{i}$ are the mean and the standard deviation estimated from the local histogram. The use of local windows is proposed to improve the adaptation of the threshold estimation to the particular characteristics of the subimage within each window. However, the $M \times M$ size of the local

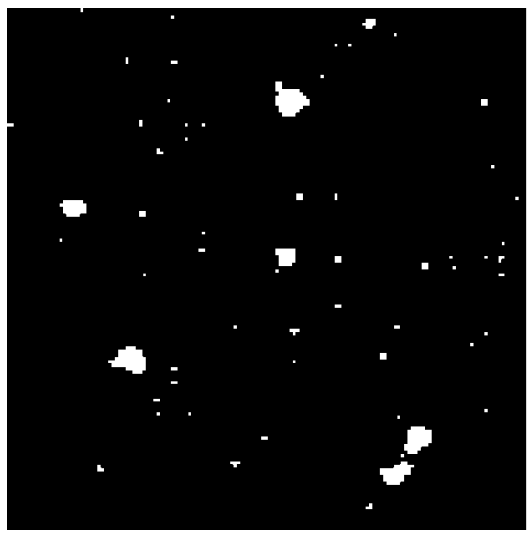

Fig. 7 Part of the processed image after segmentation by general binarization.

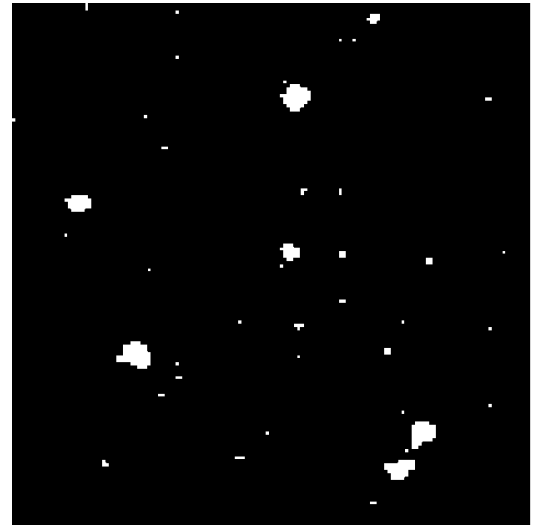

Fig. 8 Part of the processed image after segmentation by local binarization with size of window $M=64$.

window must be large enough to maintain the assumption of a local histogram with a unique Gaussian mode corresponding to the background.

The binarization of the whole image based on local thresholding is carried out by taking displacements of the window of $M / 2$ pixels in the horizontal and vertical directions. To binarize the overlapping zones, we use the logical operator AND, so that a pixel is eventually assigned as pilling if its gray level is higher than all the local thresholds $U_{i}$ of the windows that contained it. This overlapping of adjacent windows in the local binarization ensures a smooth transition between local thresholds of neighboring windows. Figure 8 shows the result of applying a local binarization with a window size of $M=64$. It can be seen that big pills are quite similar in both Figs. 7 and 8 , but there are more small objects (probably corresponding to noise) in Fig. 7 (general binarization) than in Fig. 8 (local binarization). Local binarization yields pills with a slightly smaller area that, as can be seen later, fit better the actual area.

\subsection{Reduction of Binary Noise}

In the binary image of Fig. 7, and even in Fig. 8, there are a number of very small objects contributing to the total area of pilling. However, most of these objects are too small to correspond to real pills and it is more likely that they are residual noise that should be removed.

As a first rough approach, we consider that actual pills should have a minimum size (in pixels) of at least the same order of thickness as the threads weft in the fabric. Threads are composed of some twisted bunches of thin fibers, and pills are formed by the fibers that, due to friction, migrate to the external part of the yarns and become entangled on the web surface. It is a reasonable assumption that a small accumulation of fluff or a small formed pill should be of at least the same size as the thread thickness. With this assumption, we establish a threshold of pill area $t$ that is the number of pixels covering the minimum area of an object to be considered a pill. Objects in the binarized image $I_{p}^{\prime \prime \prime}$ with area smaller than the threshold $t$ are considered as noise and therefore are neglected when computing the total area of pilling. 
At this point, we are aware of the difficulty of finding a consistent and robust threshold of pill area $t$. There are several control parameters $(k, N, M)$ previously introduced into the process. Slight variations in the values of these parameters could require a different value for the threshold of the pill area as well. As a hypothesis, if the final result obtained after applying the process (i.e., the assignment of a degree of pilling to a sample) depended critically on the value of the threshold of the pill area, this would be a weak point of our automatic method of image analysis. We seek robust solutions in which the values of parameters and thresholds are not critical and can be kept fixed, independently of potentially varying conditions. In Sec. 3 we turn back to this idea and discuss the results obtained for the Zweigle KG-741 set of standard images.

\subsection{Degree of Pilling versus Area of Pilling}

The total area of pilling $A_{p}$ can be estimated by:

$A_{p}=\sum_{T_{i}>t} T_{i}$

where $T_{i}$ is the area of the $i$ 'th pill in pixels and $t$ is the threshold of the pill area. The greater the amount and size of pills, the larger the total area of pilling $A_{p}$ and consequently the higher the degree of pilling $p$.

We applied the whole process to the Zweigle KG-741 set of standard images, which gives a reference for the degrees of pilling, from $p=1$ (no pilling) to $p=5$ (very severe pilling). We estimated the total area of pilling $A_{p}$ for each standard image of degree $p$. As expected, $A_{p}$ values increased in parallel with $p$ values.

We then compared the results of this method with the results from measuring the pilling area in the standard images by human observers. In our experiment, seven nontrained observers looked individually at the digital images of the standard sequentially displayed on a TV monitor in random order. The observers were not informed about the degree of pilling corresponding to the image they were looking at. Assisted by computer, they classified pixel by pixel, so as to separate those pixels belonging to pilling from those belonging to background in each image. Then, for each standard image of degree of pilling $p$, a set of seven segmented images provided by observers was obtained. From this set we synthesized seven additional binary images: one of them by setting as pilling all those pixels that had been classified as pilling by one or more observers (which is equivalent to OR-ing the images of the set), one by setting as pilling all those pixels that had been classified as pilling by two or more observers and so on, until reaching the image obtained by setting as pilling all those pixels that had been classified as pilling by all seven observers (which is equivalent to AND-ing the images of the set). This process of image generation was repeated for all the standard images with different degrees of pilling. The area of pilling $A_{p}^{\prime}$ assigned to each synthesized image is equal to the number of pixels of its pilling class. The resulting values $A_{p}^{\prime}$ are used as entries in the following study of the human performance of pilling segmentation.

In general, it is empirically found that the greater the magnitude of a visual stimulus, the greater the size of the
Table 3 Data of the linear regression fits of Eq. (7) with entries of the areas of pilling $A_{p}^{\prime}$ in the synthesized images obtained from the human estimations for each standard image. For the last row, data of the linear regression fit of Eq. (8) with entries of the areas of pilling $A_{p}$ obtained by the method of image analysis applied to each standard photograph.

\begin{tabular}{lrrr}
\hline \hline \multicolumn{1}{c}{ Pilling Detection } & $m$ & $A_{1}$ & $r$ \\
\hline At least by one observer (OR) & 1.00 & 343 & 0.994 \\
At least by two observers & 1.00 & 248 & 0.994 \\
At least by three observers & 1.20 & 124 & 0.988 \\
At least by four observers & 1.23 & 94 & 0.987 \\
At least by five observers & 1.30 & 64 & 0.988 \\
At least by six observers & 1.30 & 51 & 0.988 \\
All seven observers (AND) & 1.33 & 35 & 0.990 \\
By the method of image analysis & 1.00 & 165 & 0.993 \\
\hline \hline
\end{tabular}

increment that must be added to the stimulus magnitude to be just noticeable. This is mathematically stated in Weber's law which leads to the Fechner law that relates the sensation magnitude to a logarithmic function of the stimulus magnitude. ${ }^{15}$ For this reason, we considered that the degree of pilling (sensation magnitude) could be related to a logarithmic function of the area of pilling (stimulus magnitude). Moreover, the relative ease with which a human observer perceives an amount double or half something led us to consider a logarithm in base two. In the particular case of the standard image of the degree of pilling $p=1$ (no pilling), it must be taken into account that all the observers in our experiment provided the same result, for which no pixels were classified as pilling. This fact implies that the logarithmic law is not completely fulfilled, particularly for low values of area of pilling. To overcome the situation we propose to introduce a constant-which we call offset- to add to the values of segmented area of pilling. In fact, it could be said that the effect is similar to that caused by an offset mechanism in the visual perception of the pilling area in the standard images. From these considerations, we study a logarithmic approach between the degree of pilling $p$ and the observed area of pilling $A_{p}^{\prime}$. The approach can be expressed by the formula:

$p-1=\log _{2}\left(\frac{A_{p}^{\prime}+A_{1}}{A_{1}}\right)^{1 / m}$,

where $m$ is a real constant and $A_{1}$ is the area of pilling corresponding to the degree of pilling $p=1$, which we consider analogous to the offset value. A plausible explanation of the fact that all observers assign a 0 area of pilling to the standard image with $p=1$ is they calibrated themselves to fix the zero level of area of pilling to the sample known $a$ priori as the nonpilled sample. This calibration entails the existence of an offset in their gradation of the area of pilling. We performed fits by linear regression of Eq. (7) using the values $p=1, \ldots, 5$ and $A_{p}^{\prime}$ of the images synthesized from the observers' results. Table 3 presents the values of $m, A_{1}$ and the correlation coefficient $r$ for each set of synthesized calculations based on the observers' results (from OR to AND). Figure 9 shows all the fitted lines and the empirical 


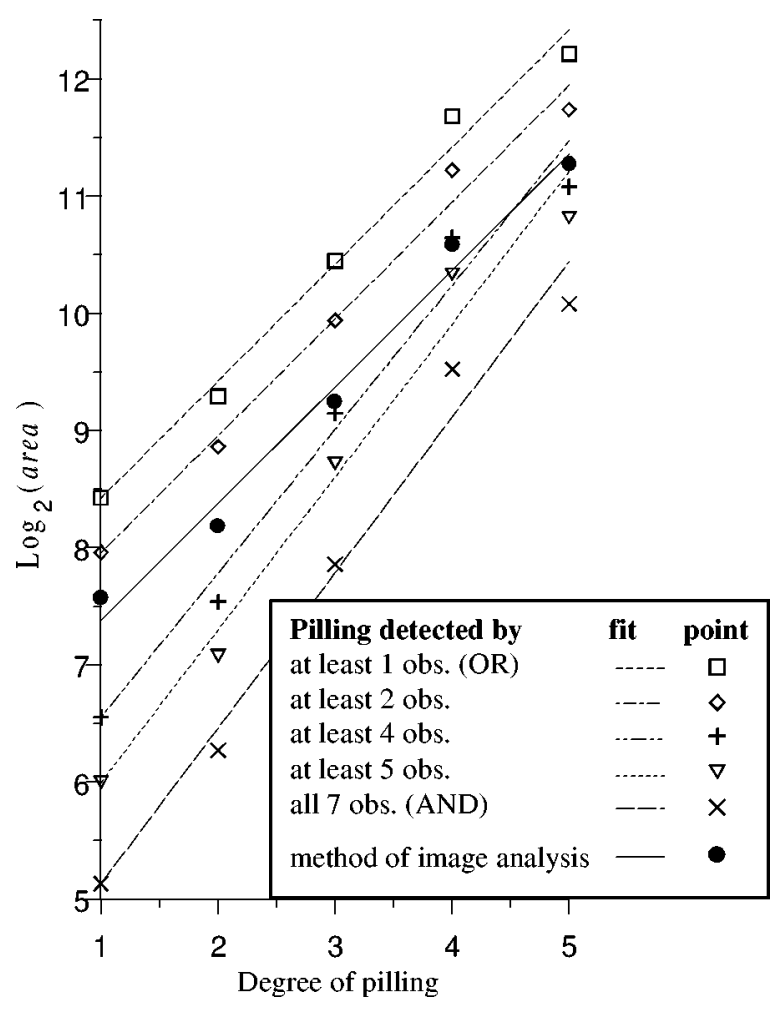

Fig. 9 Areas of pilling corresponding to the standard images of degrees $p=1, \ldots, 5$. Estimations obtained from human visual performances and from the method of image analysis. Linear regression fits of each set of points according to Eqs. (7) and (8) (Table 3).

points on a graph of $\log _{2}$ (area) versus the degree of pilling $p$. In this case, the variable area is the addition area $=A_{p}^{\prime}$ $+A_{1}$. Note that $m$ corresponds to a slope value in Fig. 9 . For $p=1$, the points represented at the lines obtained from observers' results, reach the fitted values $\log _{2}\left(A_{1}\right)$. From the values of the correlation coefficients $r$ in Table 3, all of them higher than 0.95 , we conclude that the logarithmic approach represented by Eq. (7) is fully consistent with the empirical results obtained from the observers. Moreover, a value of the slope $m$ around unity, particularly in the region of good recognition capability (objects detected by at least one observer or two observers), confirms the logarithm in base two.

For the sake of comparison with human performance, we also present in Fig. 9 the values of $\log _{2}$ (area) from the pilling areas $A_{p}$ obtained by the method of image analysis with the parameters $k=3.5, N=4, M=64, t=5$ (the thread thickness occupied 4 pixels on average) for each standard image. The variable area for these points is just $A_{p}$ because the algorithm does not have an offset mechanism, as human observers seem to have. Using linear regression we fit the five points obtained by image analysis according to the expression:

$p-1=\log _{2}\left(\frac{A_{p}}{A_{1}}\right)^{1 / m}$.

The argument of the logarithmic function of Eq. (8) differs in unity from that of Eq. (7). Apart from this, both Eqs.
(7) and (8) are similar. The result of the fit of Eq. (8) is also contained in Table 3 and graphed in Fig. 9. The correlation coefficient is $r=0.993$ and the slope is $m=1.00$. From this result, we can say that the performance of the method of image analysis is on a par with that of observers (by adding $A_{1}$ to $\left.A_{p}^{\prime}\right)$.

A feature we consider very useful for an automatic method is robustness against slight variations in the control parameters. This means that the assignment of a degree of pilling to a sample must be a solid operation, not critically dependent on given values of the parameters. To this end, we revise the correlation coefficient $r$ and the slope value $m$ as quality criteria of the fits obtained for different sets of control parameters $(k, M, t)$. Remember that the control parameter $N$ was previously checked and optimized, and $N$ $=4$ was kept constant in Sec. 2.3. A good fit should yield values of both $r$ and $m$ close to unity. Robustness is checked by comparing the values $r$ and $m$ obtained after variations in the parameters $k, M$ and $t$ within the ranges guessed previously, i.e., $k \in[2.5,4.5], \quad M$ $\in\{512$ (image size) $, 256,128,64,32,16\}, t \in[3, \ldots, 7]$. Figure 10 shows the values of the correlation coefficient $r$ in five diagrams, Figs. 10(a) to 10(e), corresponding to the different values of the window size $M$ used for binarization. In each diagram, the origin is placed in the upper left corner, the vertical axis represents the constant $k$ used to filter out periodic frequencies, and the horizontal axis represents the value of the threshold of pill area $t$. The values reached by the correlation coefficient $r$ for the different parameters $k, M$, and $t$ are displayed in gray levels in Fig. 10. The darker the cell, the higher the value of $r$. The maximum value of $r$ presented in Fig. 10 is $r=0.996$, which corresponds to $k=3.5, M=64$, and $t=6$.

The logarithmic approach can be considered for a robust performance of the method because a correlation coefficient of $r>0.95$ is obtained for a large range of values of $k$, $M$, and $t$. The window size is not a critical parameter, since the results do not change drastically among the different diagrams of Fig. 10. However, we can see that a local binarization with an appropriate window size of about 64 $\times 64$ pixels yields most of the best fits and is therefore preferable to a general binarization [see Figs. 10(d) and 10(a)]. For a window size $M=32$ and smaller, results are worse than for $M=64$. As we said in Sec. 2.3, the Gaussian model of the background becomes quite imprecise for local windows with small areas. An additional drawback of using small local windows is the increase in computing time.

A general overview of the diagrams of Fig. 10 reveals a certain correlation between the parameter $k$ of the periodic pattern mask and the threshold of pill area $t$. Low values of $k$, which mean stronger filtering of frequencies, mainly in the low frequencies spectrum area, tend to eliminate more uninteresting information. Consequently, these low $k$ values only require a low value of the threshold of pill area $t$, i.e., objects with an area of only very few pixels should be considered noise and need to be suppressed to obtain a good fit ( $r$ high). On the contrary, higher values of $k$, which entail a lighter filtering of low frequencies, require higher values of $t$ (objects with area consisting of more pixels should be considered noise and be eliminated) to reach a fitted line with a similar correlation coefficient $r$ as before. 


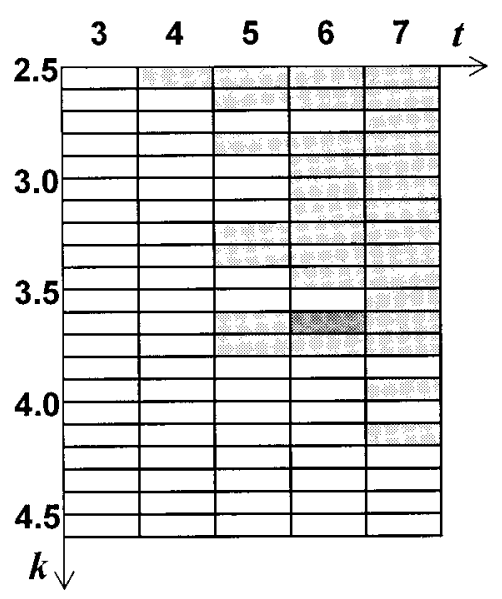

(a) $\mathrm{M}=512$

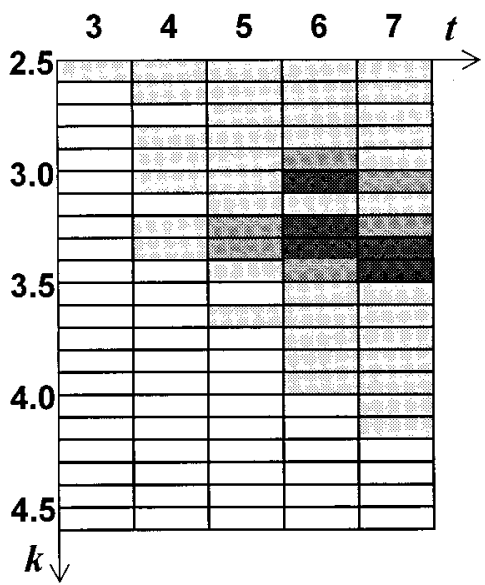

(b) $\mathrm{M}=256$

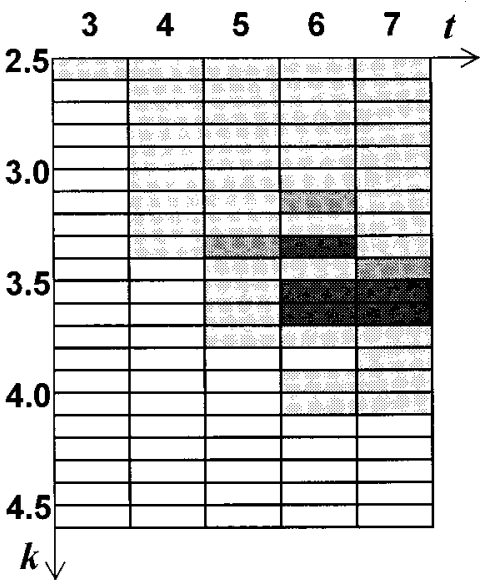

(c) $\mathrm{M}=128$

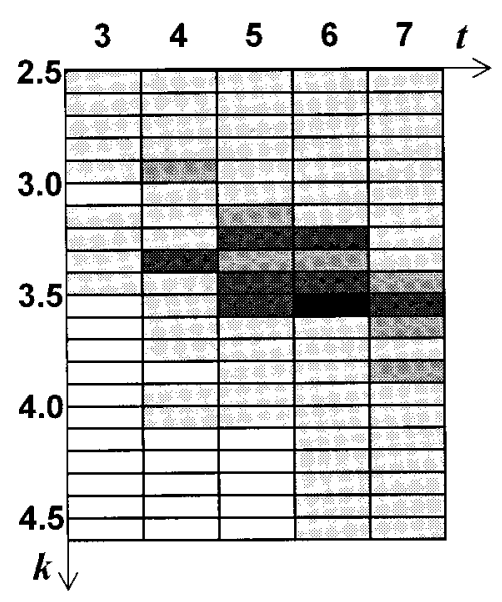

(d) $M=64$

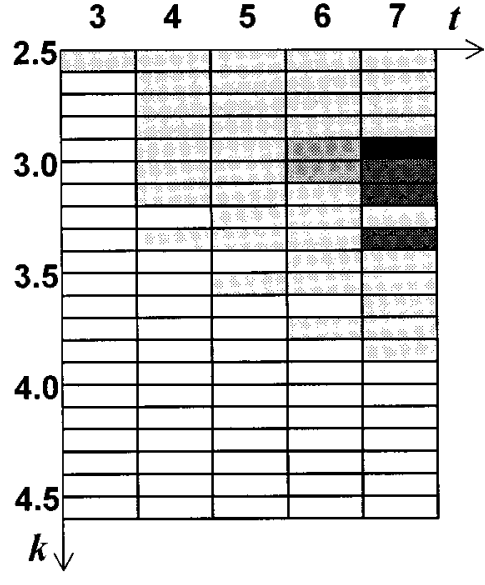

(e) $\mathrm{M}=32$

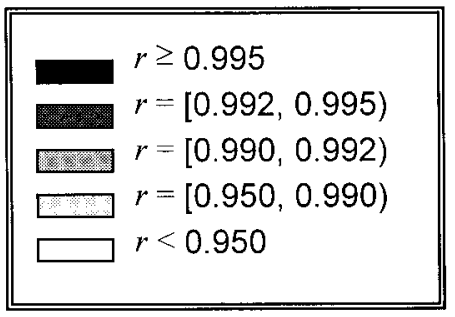

Fig. 10 Correlation coefficients $r$ of the linear regression fits of Eq. (8), taking the values $A_{p}$ provided by the method of image analysis with the control parameters $k, M$, and $t$ varying in $k=[2.5,4.5], M$ $=[512,32]$, and $t=[3,7]$.

We calculated the value of the slope $m$ in all the cases considered in Fig. 10. To shorten the presentation of results, Fig. 11 contains only the diagram corresponding to the selected window size $M=64$. Similar diagrams are obtained for the other values of $M$ and are therefore not presented here. The diagram of Fig. 11 is organized in the same way as that in Fig. 10. The values reached by the slope $m$ for the different parameters $k$ and $t$ are displayed in gray levels again. The darker the cell, the closer the values of $m$ to unity. The closest to unity is $m=1.00$, which corresponds to $k=3.5$ and $t=5$. The $m$ values close to unity are distributed along the diagonal in Fig. 11 more clearly than in Fig. 10. It again shows the correlation between parameters $k$ and $t$.

There is good overall agreement between the diagrams in Figs. 10(d) and 11, which suggests that the best fits to the logarithmic law correspond to a slope value $m$ very close to unity. Taking into account both Figs. 10(d) and 11, we choose the pair $(k, t)=(3.5,5)$ that reaches the values of $r=0.993$ with $m=1.00$.

As a result of this analysis, we fix the best control parameters with the values $k=3.5, N=4, M=64$, and $t=5$, although we have already demonstrated that the exact values are not critical.

\section{Discussion}

In the previous section we presented an automatic method for pilling evaluation by image analysis and showed the highly promising results obtained after applying it to standard images. The accuracy achieved with this method enabled us to approach the underlying rule- unknown to usused by experts to elaborate the standard. Our approach, described by Eqs. (7) and (8), assumes a logarithmic in 


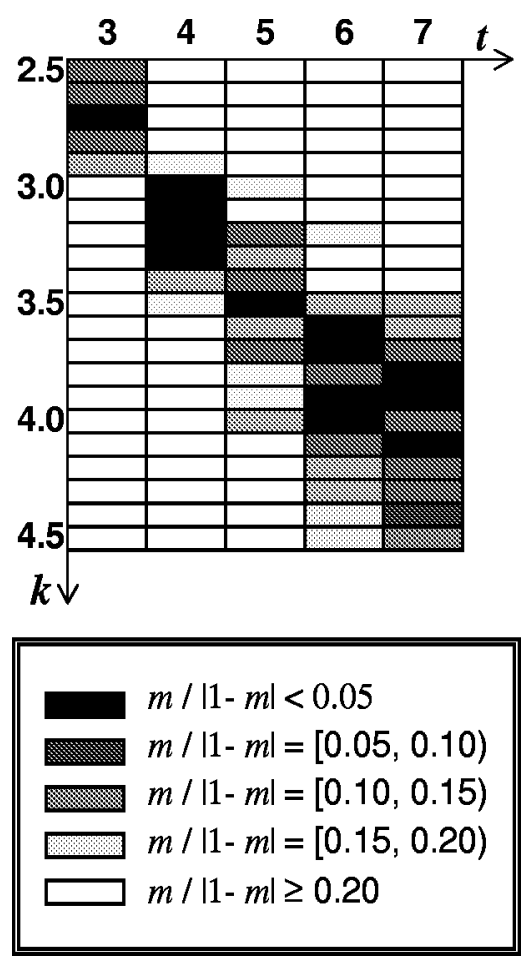

Fig. 11 Slope $m$ of the linear regression fits of Eq. (8) with the values of $A_{p}$ provided by the method of image analysis with the control parameters $M=64$ and $k$ and $t$ varying in $k=[2.5,4.5]$ and $t=[3,7]$.

base two relationship between the area of pilling and the degree of pilling. This assumption is consistent with the related logarithmic law ${ }^{15}$ which characterizes human visual perception. In fact, a comparison of the results obtained by the method with those obtained by a group of nontrained observers, who performed the segmentation by visual inspection of the standard images, demonstrated the feasibility of the logarithmic approach. We then tested the robustness of the method against slight variations in the values of the control parameters. The results of our analysis confirm the robustness of the method. The values of the control parameters are not critical and slight variations in them within reasonable ranges do not severely alter the method's performance.

Nevertheless, other approaches different from the logarithmic one studied here could be considered and analyzed. From the estimations of the pilling area of the standard images carried out by the visual inspection of a group of observers, another empirical law, different from Eq. (7), could be fitted. For instance, we may fit the $A_{p}^{\prime}$ values of each set of the images synthesized from the observers' responses according to the expression:

$A_{p}^{\prime}=C(p-1)^{3} \exp \left[-\left(\frac{p-1}{\alpha}\right)^{2}\right]$

where $C$ and $\alpha$ are real constants. Taking the $A_{p}$ values obtained by the method of image analysis, however, we can fit them according to the expression:

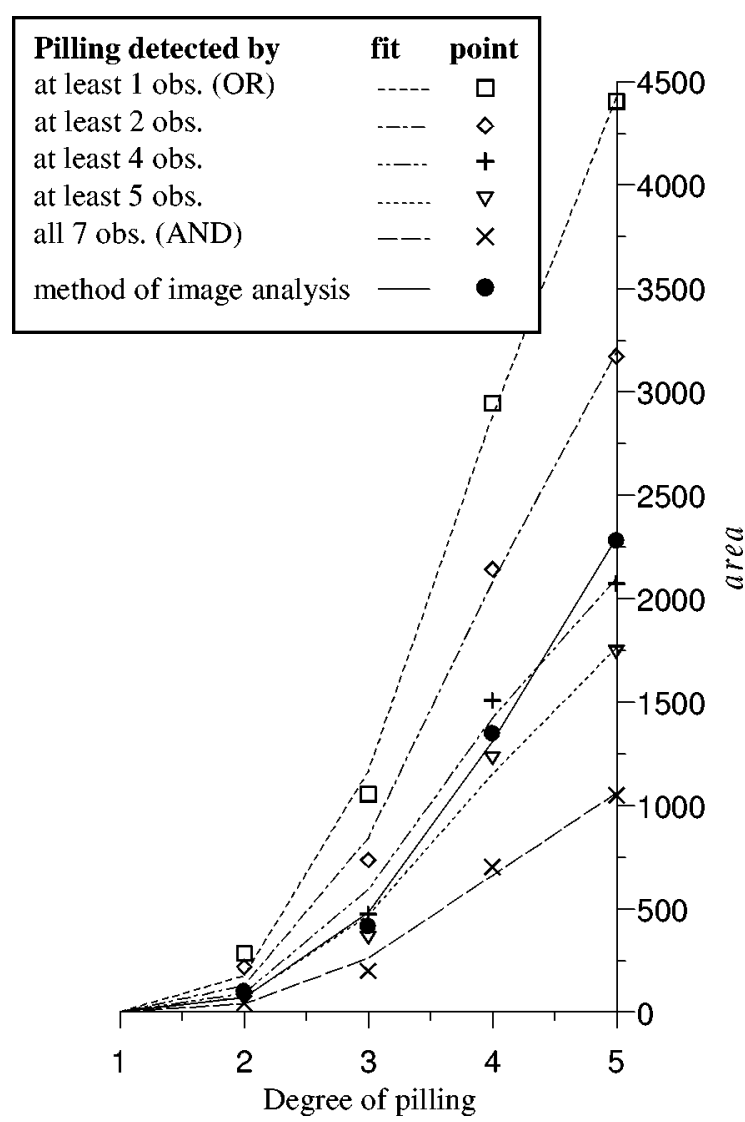

Fig. 12 Areas of pilling corresponding to the standard images of degrees $p=1, \ldots, 5$. Estimations obtained from human visual performances and from the method of image analysis. Fits of each set of points according to Eqs. (9) and (10) (Table 4).

$A_{p}-A_{1}=C(p-1)^{3} \exp \left[-\left(\frac{p-1}{\alpha}\right)^{2}\right]$.

Note that the offset value is not taken into account in Eq. (9), but in Eq. (10), where the area of pilling $A_{1}$ obtained by the method for the degree $p=1$ is subtracted from the area of pilling $A_{p}$. This enables $A_{p}^{\prime}$ and $A_{p}-A_{1}$ to be compared. In Fig. 12 the experimental points and the fits of the area of pilling versus the degree of pilling area are graphed. Equation (9) was used to fit the results of the observers, whereas Eq. (10) was used to fit the results obtained by the method. Table 4 contains the values of $C$ and $\alpha$ yielded by each fit, together with its correlation coefficient $r$. The correlation coefficient of the fit corresponding to the method $r=0.999$ is equal to the best obtained from the observers' data. However, the value of $\alpha=4.80$ with the method differs a little from the values of $\alpha$ obtained with the observers.

The analyses of Table 4 and Fig. 12 show that even more accurate fits (higher $r$ ) are achieved with this second approach than with the previous logarithmic one. This result does not mean that the logarithmic approach must be rejected. The correlation coefficients reached under the logarithmic assumption were reasonably good (see Table 3 ), though not as high on average as those obtained using the second approach [represented by Eqs. (9) and (10)]. In 
Table 4 Data of the fits of Eqs. (9) and (10) with entries of the areas of pilling obtained from human calculations $A_{p}^{\prime}$ and from the method of image analysis $A_{p}$, respectively.

\begin{tabular}{lrrr}
\hline \multicolumn{1}{c}{ Pilling Detection } & $C$ & $\alpha$ & $r$ \\
\hline At least by one observer (OR) & 186.20 & 4.01 & 0.999 \\
At least by two observers & 134.56 & 4.01 & 0.998 \\
At least by three observers & 115.75 & 3.88 & 0.998 \\
At least by four observers & 96.84 & 3.84 & 0.997 \\
At least by five observers & 74.72 & 3.99 & 0.996 \\
At least by six observers & 61.32 & 3.93 & 0.994 \\
All seven observers (AND) & 40.39 & 4.23 & 0.997 \\
By the method of image analysis & 71.55 & 4.80 & 0.999 \\
\hline \hline
\end{tabular}

fact, both approaches led to a similar correspondence between the area of pilling and the degree of pilling in the interval of $p=1, \ldots, 5$. Thus, these results confirm that the empirical approach sought, and so the formula, do not necessarily have to be unique to describe the process.

Furthermore, the robustness of our method is demonstrated not only through the noncritical values of the control parameters (Sec. 2.5), but also through a noncritical mathematical description of the underlying law. Hence, because which mathematical expression is chosen is not critical, we decided to use the logarithmic approach. Although the second approach reached slightly higher correlation coefficients, the logarithmic approach is consistent with the mentioned visual perception laws and covers better the performance of the mechanism. Furthermore, the logarithmic approach makes it easy to optimize the values of the control parameters $(k, M, t)$ introduced in the algorithm within their tolerance ranges, as shown in Sec. 2.5.

Since neither the values of the parameters nor the mathematical expression relating the area of pilling to the degree of pilling are actually critical, the selection of the values $k=3.5, M=64, N=4$ and $t=5$ for the control parameters of the algorithm is considered valid.

\section{Conclusions}

We developed a method of digital image analysis for the objective and automatic evaluation of pilling in wear-andtear fabrics. The method calculates the total area of pilling in the sample image. It involves operations in both the spatial and frequency domains to achieve an efficient segmentation of pills from the textured background of the web. The algorithm discriminates the degrees of pilling of the standard set and provides highly satisfying results. No control parameter introduced in the algorithm has a critical value whose variation in a reasonable range alters the result drastically. This demonstrates the robustness of the method for any slight variation in the control parameters.

Two empirical approaches to establishing a correspondence between the area of pilling in the standard images and the degree of pilling were analyzed. Both approaches, formulated according to the calculations of the area of pilling by visual inspection, reach acceptable degrees of precision and establish a similar correspondence between the area of pilling and the degree of pilling in the studied intervals. Although the logarithmic approach did not achieve the most precise fits, it was convincing because it is consistent with the well-known logarithmic laws that describe human visual perception. In addition, the formula corresponding to this logarithmic approach is neat and comprehensible.

The results obtained by the method of image analysis are highly consistent with those obtained by the observers, when it is remembered that all the observers assigned an area of zero pilling to the standard image of degree $p=1$.

The fits of the results provided by the method according to each of the formulas representative of the two approaches reach high values in their correlation coefficients. The logarithmic approach is the best for the method and shows the easiest way to determine the best (but noncritical) values for the control parameters.

Note that the task of formulating the correspondence between the area of pilling and the degree of pilling defined by the standard images is not a requirement for an objective extraction of features such as the area of pilling, but for the comparison with the results provided by experts. With this correspondence, we set the basis for a future validation of the method with respect to experts when operating on real samples.

\section{Acknowledgments}

We acknowledge the Comisión Interministerial de Ciencia y Tecnología (CICYT), under project No. TAP97-1089.

\section{References}

1. R. H. Brand and B. M. Bohmfalk, "A mathematical model of pilling mechanisms,', Text. Res. J. 37, 467-476 (1967).

2. W. Wegener and B. Wulfhorst, "Pilling an textilen Flächengebilden," Textilbetrieb 103(4) 39-51 (Set. 1985).

3. R. B. Ramgulam, J. Amirbayat, and I. Porat, "The objective assessment of fabric pilling. Part I: methodology,' J. Text. Inst. 84(2), 221-226 (1993).

4. D. Brzakovic, H. Beck, and N. Sufi, "Flaw detection and classification in texture materials" in Advances in Image Analysis, Y. Mahdavieh and R. C. González, Eds., pp. 244-274, SPIE, Bellingham, WA (1992).

5. M. S. Millán and J. Escofet, "Fourier domain based angular correlation for quasiperiodic pattern recognition. Applications to web inspection,' Appl. Opt. 35(31), 6253-6260 (1996).

6. C. Ciamberlini, F. Francini, G. Longobardi, P. Sansoni, and B. Tiribilli, "Defect detection in textured materials by optical filtering with structured detectors and self-adaptable masks,' Opt. Eng. 35(3), 838844 (1996)

7. W. J. Jasper, S. J. Garnier, and H. Potlapalli, “Texture characterization and defect detection using adaptive wavelets,' Opt. Eng. 35(11), 3140-3149 (1996)

8. B. Xu, "Identifying fabric structures with fast Fourier transform techniques,"' Text. Res. J. 66(8), 496-506 (1996).

9. A. Konda, L. C. Xin, M. Takadera, Y. Okoshi, and K. Toriumi, "Evaluation of pilling by computer image analysis,' J. Text. Mach. Soc. Jpn. 36(3), 96-107 (1990).

10. H. C. Abril, M. S. Millán, and R. Navarro, "Pilling evaluation in fabrics by digital image processing,' in Vision Systems: Applications, P. A. Kammenos and B. Nickolay, Eds., Proc. SPIE 2786, 19-28 (1996).

11. H. C. Abril, M. S. Millán, Y. Torres, and R. Navarro, "Image segmentation based on a Gaussian model applied to pilling evaluation in fabrics,', in New Image Processing Techniques and Applications: Algorithms, Methods, and Components II, R. J. Ahlers and P. Réfrégier, Eds., Proc. SPIE 3101, 283-291 (1997).

12. E. R. Douguerty, An Introduction to Morphological Image Processing, p. 120, SPIE Press, Bellingham WA (1992).

13. J. D. Gaskill, Linear System, Fourier Transform, and Optics, pp. 489-491, John Wiley, New York (1978).

14. R. Navarro, J. Santamaría, and R. Gómez, "Automatic log spectrum restoration of atmospheric seeing,' Astron. Astrophys. 174, 344-351 (1987).

15. G. Wyszecky and W. S. Stiles, Color Science: Concepts and Methods, Quantitative Data and Formulae, 2nd ed., pp. 489-491, John Wiley $\&$ Sons, New York (1982). 


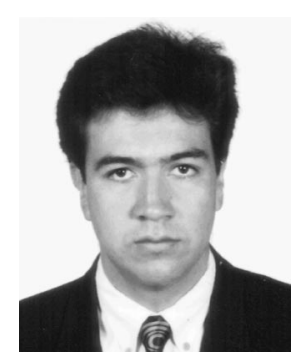

Héctor C. Abril graduated from the Universidad Industrial de Santander, Colombia, in 1993 with a BS degree in systems and computer engineering. He received his MS degree in 1996 and he is currently working toward his $\mathrm{PhD}$ degree in applied optics at the Department of Optics, Universitat Politècnica de Catalunya, Spain. His research interest is digital image processing. Particularly, he is interested in quality control through digital imaging, in the textile industry.

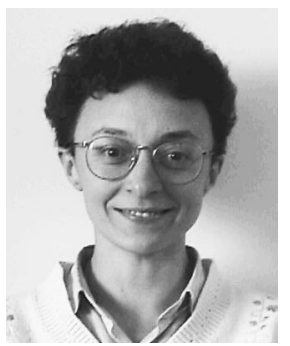

María S. Millán received the BS and MS degrees in physics from the Universitat de València in 1984 and 1985, respectively. In 1990, she received the PhD degree in physics from the Universitat Autonoma de Barcelona. Since 1984 she has been a professor in the Department of Optics and Optometry of the Universitat Politècnica de Catalunya. Her fields of research involve pattern recognition, color, texture analysis, optical processing, and image processing. She is a member of the Sociedad Española de Optica, the European Optical Society, and SPIE.

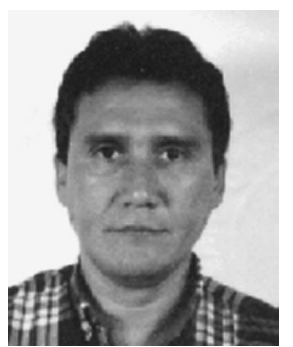

Yezid Torres obtained his $\mathrm{PhD}$ in optics and signal processing at the Université de Franche Comté, Besançon, France, in 1983. He joined the Physics School at the Universidad Industrial de Santander, Colombia in 1984 where he is Professor of physics. He spent research stays at the Laboratoire d'Optique P. M. Duffiex, Besançon, France; the Centre d'Optique, Photonique et Laser COPL, Quebec, Canada and the Laboratorio de Procesado de Imágenes, Terrassa, España. He has coauthored more than 20 papers and 40 oral presentations and holds one patent. His research activities have included signal and image processing; and Fourier optics.

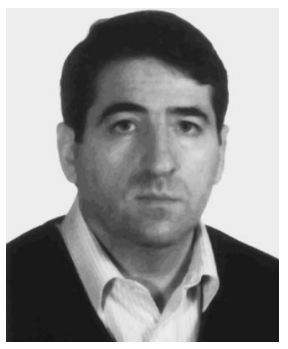

Rafael Navarro received the MS and $\mathrm{PhD}$ degrees in physics from the University of Zaragoza, Spain, in 1979 and 1984 respectively. From 1985 to 1986 he was an optical and image processing engineer at the Instituto de Astrofisica de Canarias. $\mathrm{He}$ joined the Instituto de Optica of the Consejo Superior de Investigaciones $\mathrm{Ci}$ entíficas in 1987, where he is currently a senior scientific researcher. Since 1988 he has headed the Imaging \& Vision group and since 1994 he has been associate director of the Instituto de Optica. He is interested in human vision, optics, and image processing. 